\title{
Hermaphroditism in fishes: an annotated list of species, phylogeny, and mating system
}

\author{
Tetsuo Kuwamura ${ }^{1,2} \cdot$ Tomoki Sunobe $^{3} \cdot$ Yoichi Sakai $^{4} \cdot$ Tatsuru Kadota $^{5} \cdot$ Kota Sawada $^{6}$
}

Received: 5 January 2020 / Revised: 21 April 2020 / Accepted: 21 April 2020 / Published online: 7 May 2020

(c) The Author(s) 2020

\begin{abstract}
Fewer than $1 \%$ of vertebrate species are hermaphroditic, and essentially all of these are fishes. Four types of hermaphroditism are known in fishes: simultaneous (or synchronous) hermaphroditism ( $\mathrm{SH}$ ), protandry (male-to-female sex change; PA), protogyny (female-to-male sex change; PG), and bidirectional sex change (BS or reversed sex change in protogynous species). Here we present an annotated list of hermaphroditic fish species from a comprehensive review and careful re-examination of all primary literature. We confirmed functional hermaphroditism in more than 450 species in 41 families of 17 teleost orders. PG is the most abundant type (305 species of 20 families), and the others are much less abundant, BS in 66 species of seven families, SH in 55 species of 13 families, and PA in 54 species of 14 families. The recently proposed phylogenetic tree indicated that SH and PA have evolved several times in not-closely related lineages of Teleostei but that PG (and BS) has evolved only in four lineages of Percomorpha. Examination of the relation between hermaphroditism type and mating system in each species mostly supported the size-advantage model that predicts the evolution of sequential hermaphroditism. Finally, intraspecific variations in sexual pattern are discussed in relation to population density, which may cause variation in mating system.
\end{abstract}

Keywords Bidirectional $\cdot$ Protandry $\cdot$ Protogyny $\cdot$ Sex change $\cdot$ Simultaneous hermaphroditism

Electronic supplementary material The online version of this article (https://doi.org/10.1007/s10228-020-00754-6) contains supplementary material, which is available to authorized users.

Tetsuo Kuwamura

kuwamura@lets.chukyo-u.ac.jp

1 School of International Liberal Studies, Chukyo University, Yagoto-honmachi, Nagoya 466-8666, Japan

2 Present Address: Faculty of Liberal Arts and Sciences, Chukyo University, Yagoto-honmachi, Nagoya 466-8666, Japan

3 Laboratory of Fish Behavioral Ecology, Tateyama Station, Field Science Center, Tokyo University of Marine Science and Technology, Banda, Tateyama 294-0308, Japan

4 Graduate School of Integrated Sciences for Life, Hiroshima University, Kagamiyama, Higashi-Hiroshima 739-8528, Japan

5 Seikai National Fisheries Research Institute, Japan Fisheries Research and Education Agency, Taira-machi, Nagasaki 851-2213, Japan

6 Oceanic Ecosystem Group, National Research Institute of Far Seas Fisheries, Japan Fisheries Research and Education Agency, Fukuura, Kanagawa 236-8648, Japan

\section{Introduction}

Over one-half of the world's living vertebrates $(>60,000$ species) are fishes (>32,000 species; Nelson et al. 2016). Approximately $99 \%$ of all vertebrate species consist of separate-sex individuals (gonochorists), i.e., pure males and pure females. The other $1 \%$ of vertebrate species are hermaphroditic, and almost all of them are fishes (Avise 2011; Ashman et al. 2014). Among hermaphroditic fishes, four major types of hermaphroditism are known (Sadovy de Mitcheson and Liu 2008; Munday et al. 2010): simultaneous (or synchronous) hermaphroditism ( $\mathrm{SH})$, protandry (PA), protogyny (PG), and bidirectional sex change (BS or reversed sex change in protogynous species; Kuwamura et al. 2014). Simultaneous hermaphrodites produce both mature eggs and sperm in their gonads at the same time, but self-fertilization rarely occurs in fishes (Avise 2011). In sequential hermaphrodites, either male-to-female sex change (PA) or female-tomale sex change (PG) occurs during the lifetime of an individual. Reports on bidirectional sex change within a species have increased since the 1990s (Kuwamura and Nakashima 1998; Munday et al. 2010), mostly as reversed sex change in 
protogynous species when observed in the field (Kuwamura et al. 2014, 2016a).

The evolution of hermaphroditism among animals has been explained by two major hypotheses: the low-density model for simultaneous hermaphroditism and the sizeadvantage model for sequential hermaphroditism (Ghiselin 1969, 1974). In low-density conditions, few mating opportunities are expected, and mating success will be much higher in simultaneous hermaphrodites than that in gonochorists if random mating occurs (Tomlinson 1966), although hermaphrodites suffer the energetic cost of maintaining two reproductive systems (Heath 1977). Self-fertilization further increases the benefits of simultaneous hermaphroditism under extreme low density with little opportunity to find conspecifics (Tomlinson 1966). In addition, simultaneous hermaphroditism is presumably favored by diminishing fitness return for the investment to one sexual function, e.g., owing to small mating groups or brood space limitation (Charnov 1982).

For sequential hermaphroditism, the size-advantage model (SA model) predicts that the occurrence and direction of sex change are determined by mating system, because the relation between reproductive success and body size of males depends on mating system, while reproductive success of females increases with growth irrespective of mating system (Warner 1975, 1984). For example, in species with random mating regardless of male body size, in which females do not have preference for large males and accept small ones, male reproductive success is not related to body size, and male-to-female sex change (PA) will be favored, because the reproductive success of small males is higher than that of females of the same size, and vice versa in large males and females. By contrast, in species with polygynous mating systems, in which large males monopolize females, male reproductive success sharply increases with growth, and female-to-male sex change (PG) will be favored, because large females can increase reproductive success by changing sex to male. Contrastingly, in species with sizeassortative mating or group spawning by one female with multiple males (i.e., intense scramble-type sperm competition), in which reproductive success of males and females increases with growth in a similar fashion, gonochorism will evolve because of the cost of sex change (Warner 1975, 1984). Some mating systems with extensive sperm competition and size-fecundity skew will also reduce or eliminate the size advantage and result in a reduction or even a lack of sex change (Muñoz and Warner 2003, 2004). Bidirectional sex change has recently been regarded as a tactic to secure mating opportunities when mate-search (finding an oppositesex individual) after the loss of partners is difficult or costly because of low density, and it is suggested to have derived from either protogynous (Kuwamura et al. 2014, 2016a) or gonochoristic ancestors (Sunobe et al. 2017). Large amounts of evidence supporting the SA model have been reported, especially from coral reef fishes (Nakazono and Kuwamura 1987; Warner 1988; Munday et al. 2006; Sadovy de Mitcheson and Liu 2008; Kazancığlu and Alonzo 2010; Erisman et al. 2013). However, the mating systems of many other hermaphroditic fishes are still unknown.

Sadovy de Mitcheson and Liu (2008) published a comprehensive review of functional hermaphroditism in teleost fishes, providing family accounts with a genus-level list of 94 genera in 27 families of seven teleost orders (based on the classification of Nelson 2006) with confirmed functional hermaphroditism. They also listed 31 genera in 21 teleost families that have been suggested as hermaphroditic but not confirmed. Hermaphroditism has evolved in many different fish lineages, predominantly among tropical marine perciforms, in which hermaphroditism expression diversity is the greatest. However, because the sexual pattern and mating system are often highly variable even within a single genus or species (Munday et al. 2006), the genus-level list provided by Sadovy de Mitcheson and Liu (2008) is not sufficient to estimate the evolutionary patterns of sexuality. Although Devlin and Nagahama (2002) provided a list of 259 species in 25 teleost families, they did not make such a clear distinction between functional and non-functional hermaphroditism which is essential to evolutionary/ecological analyses.

The purpose of this study is to present an annotated species-level list of functional hermaphroditism in fishes, which will enable a wide range of interesting analyses, after a comprehensive review and careful re-examination of all primary literature. Frequencies of hermaphroditism in each family and order were summed up, and its distribution on the recently proposed phylogenetic tree (Betancur-R et al. 2017) was examined. Mating systems of hermaphroditic fish species were also reviewed, and the relation between the type of hermaphroditism in each species and its mating system was investigated to test the SA model. Finally, intraspecific variations in sexual pattern are discussed in relation to population density, which may cause variation in mating system.

\section{Materials and methods}

Construction of the database of hermaphroditic fishes. We searched original papers on hermaphroditic fish species using references of review papers (e.g., Nakazono and Kuwamura 1987; Devlin and Nagahama 2002; Sadovy de Mitcheson and Liu 2008) and by Internet searches (mainly by Google Scholar) entering keywords such as hermaphroditism, sex change, protogyny, and protandry along with names of known hermaphroditic taxa (orders/families/genera). For each species, we recorded type of hermaphroditism (SH, PA, PG, and BS), methods of confirmation (histology, aquarium or field observations, see below), mating system 
(see below), habitat (e.g., deep sea, coral reef), reference, and remarks. Species name and habitat are based on FishBase (https://www.fishbase.in). The results were compiled into the list based on the fish classification system of Nelson et al. (2016). Then, to avoid overlooking any species, we conducted similar Internet searches for papers reporting hermaphroditism in each of the remaining families of fishes without known cases of hermaphroditism.

Table 1 is a simplified table in which only the type of hermaphroditism and mating system is shown, and detailed records and references are shown in Electronic supplementary material, Table S1 and S2, respectively. In each table, families and orders are arranged following the order of Nelson et al. (2016), and genera and species in alphabetical order within each family and genus, respectively.

To determine if hermaphroditism was confirmed or not in each species, we applied the criteria for functional hermaphroditism following Sadovy and Shapiro (1987) and Sadovy de Mitcheson and Liu (2008), i.e., detailed gonadal histological series ideally illustrated with photomicrographs that show various stages of sexual transition (as examples), simultaneous occurrence of mature testicular and ovarian tissues in gonads, or field or aquarium observations of gamete release and/or sexual characteristics (e.g., shape of urogenital papilla) in identified individuals. Moreover, histological observation on transition of gonad-associated structures could be used to determine the occurrence of sex change (Cole and Shapiro 1990; Sunobe et al. 2017), and microscopic observation of eggs and sperm is also useful to confirm sexual maturation. Species with suggested, but not confirmed functional hermaphroditism were also recorded (Table S1 with remarks, "?" indicates weak evidence), but excluded from Table 1.

Taxonomic, phylogenetic, and ecological distributions of hermaphroditism. Frequencies of each type of hermaphroditism, i.e., SH, PA, PG, and BS (reversed sex change), in each family and order were summed up and compared between marine and freshwater families. The phylogenetic tree of Betancur-R et al. (2017), which shows the relationship of major groups (ordinal or supraordinal taxa), was used to examine the distribution of each type of hermaphroditism.

Mating systems in hermaphroditic fishes. To test the SA model (Warner 1975), the relation between the type of hermaphroditism and mating system of each species was investigated. Based on the data cited in Table S1, we classified the mating systems into the following seven types (modified from Kuwamura 1997; Table 1).

1) Random mating: Females mate with males regardless of their body size, without continuing pair bond (i.e., close and lasting association between a male and female).

2) Non-size-assortative monogamy: Pair bonding of a male and a female, which has no preference for male size and accepts a smaller male.
3) Size-assortative monogamy: Pair bonding of a male and a female, in which both prefer larger mates, resulting in pairing of similar-sized mates.

4) Harem polygyny: A large male monopolizes a harem of females.

5) Male-territory-visiting polygamy: Females visit male territories to mate. Males may establish small territories during spawning time even within a multi-male group.

6) Group spawning: Spawning of a single female with multiple males occurs without continuous bonding usually in a spawning aggregation.

7) Spawning aggregation: We used this category when spawning aggregation has been reported, but the detailed mating system is unknown (e.g., neither male territory nor group spawning has been reported).

Facultative monogamy in polygamous species, which may frequently occur in low-density condition (Barlow 1984), is not shown in Table 1.

\section{Results and discussion}

Phylogeny and hermaphroditism. We confirmed functional hermaphroditism in more than 450 species of 156 genera in 41 families of 17 teleost orders (based on the classification of Nelson et al. 2016). In Table 1, species with confirmed functional hermaphroditism were listed, indicating the type of hermaphroditism ( $\mathrm{SH}, \mathrm{PA}, \mathrm{PG}$, and $\mathrm{BS}$ ). A detailed and annotated list of hermaphroditic fish species including additional information and unconfirmed species is provided as Electronic supplementary material Table S1 with a complete reference list of more than 500 papers $(\mathrm{S} 2)$. We found functional hermaphroditism in 461 species and all of them are teleosts (Infraclass Teleostei). Two types of hermaphroditism, i.e., PG and BS, are reported within a single species in 18 species (Table 1).

We found at least one hermaphroditic species in 41 families (9\% of 470 families of Teleostei; Table 2). Percentage of hermaphroditic species in a family widely varied from $0.1 \%$ ( 2 of 1762 species in Cichlidae) to $100 \%$ (in both species of Giganturidae and Eleginopsidae). Among fish families including over 30 species, the percentage was relatively high in Sparidae (84\%), Scaridae (35\%), Ipnopidae (28\%), Lethrinidae (26\%), Pomacanthidae (26\%), Labridae (19\%), Cirrhitidae (18\%), and Serranidae (17\%), although these percentages represented the lower limits because sexual patterns of all species have not always been investigated in each family.

PG was the most abundant hermaphroditism type (305 species, $66 \%$ of 461 hermaphroditic species), while other types were much less abundant, i.e., BS in 66 species (14\%), $\mathrm{SH}$ in 55 species (12\%), and PA in 54 species (12\%) (Table 2). SH, PA, PG, and BS were the most abundant in 
Table 1 List of hermaphroditic fish species and their mating systems. Order and family names are arranged following Nelson et al. (2016), and genus and species in alphabetical order within each family and genus, respectively

\begin{tabular}{|c|c|c|c|}
\hline \multicolumn{2}{|l|}{ Order } & \multirow[t]{2}{*}{ Sexual pattern } & \multirow[t]{2}{*}{ Mating system } \\
\hline Family & Species & & \\
\hline \multicolumn{4}{|l|}{ Anguilliformes } \\
\hline Muraenidae & Gymnothorax griseus & SH & \\
\hline Muraenidae & Gymnothorax pictus & $\mathrm{SH}$ & \\
\hline Muraenidae & Gymnothorax thyrsoideus & $\mathrm{SH}$ & \\
\hline Muraenidae & Rhinomuraena quaesita & PA & \\
\hline \multicolumn{4}{|l|}{ Clupeiformes } \\
\hline Clupeidae & Tenualosa macrura & PA & \\
\hline Clupeidae & Tenualosa toli & PA & \\
\hline \multicolumn{4}{|l|}{ Cypriniformes } \\
\hline Cobitidae & Cobitis taenia & PA, G & \\
\hline \multicolumn{4}{|l|}{ Stomiiformes } \\
\hline Gonostomatidae & Cyclothone atraria & $\mathrm{PA}$ & \\
\hline Gonostomatidae & Cyclothone microdon & PA & \\
\hline Gonostomatidae & Gonostoma elongatum & PA & \\
\hline Gonostomatidae & Sigmops bathyphilum & $\mathrm{PA}$ & \\
\hline Gonostomatidae & Sigmops gracile & PA & \\
\hline \multicolumn{4}{|l|}{ Aulopiformes } \\
\hline Ipnopidae & Bathymicrops brevianalis & $\mathrm{SH}$ & \\
\hline Ipnopidae & Bathymicrops regis & $\mathrm{SH}$ & \\
\hline Ipnopidae & Bathypterois grallator & $\mathrm{SH}$ & \\
\hline Ipnopidae & Bathypterois viridensis & SH & \\
\hline Ipnopidae & Bathypterois quadrifilis & $\mathrm{SH}, \mathrm{G}$ & \\
\hline Ipnopidae & Bathypterois mediterraneus & SH & \\
\hline Ipnopidae & Bathytyphlops marionae & $\mathrm{SH}$ & \\
\hline Ipnopidae & Ipnops agassizii & $\mathrm{SH}$ & \\
\hline Ipnopidae & Ipnops meadi & $\mathrm{SH}$ & \\
\hline Giganturidae & Gigantura chuni & $\mathrm{SH}$ & \\
\hline Giganturidae & Gigantura indica & $\mathrm{SH}, \mathrm{G}$ & \\
\hline Bathysauridae & Bathysaurus ferox & $\mathrm{SH}$ & \\
\hline Chlorophthalmidae & Chlorophthalmus agassizi & $\mathrm{SH}$ & \\
\hline Chlorophthalmidae & Chlorophthalmus brasiliensis & SH & \\
\hline Chlorophthalmidae & Parasudis truculenta & $\mathrm{SH}$ & \\
\hline Notosudidae & Ahliesaurus brevis & $\mathrm{SH}$ & \\
\hline Scopelarchidae & Benthalbella infans & $\mathrm{SH}$ & \\
\hline Scopelarchidae & Scopelarchus guentheri & SH & \\
\hline Paralepididae & Antopterus pharao & $\mathrm{SH}$ & \\
\hline Paralepididae & Lestidium pseudosphyraenoides & $\mathrm{SH}$ & \\
\hline Alepisauridae & Omosudis lowii & $\mathrm{SH}$ & \\
\hline \multicolumn{4}{|l|}{ Gobiiformes } \\
\hline Gobiidae & Coryphopterus alloides & PG & \\
\hline Gobiidae & Coryphopterus dicrus & PG & \\
\hline Gobiidae & Coryphopterus eidolon & PG & \\
\hline Gobiidae & Coryphopterus glaucofraenum & PG & MTV polygamy \\
\hline Gobiidae & Coryphopterus hyalinus & PG & \\
\hline Gobiidae & Coryphopterus lipernes & PG & \\
\hline Gobiidae & Coryphopterus personatus & PG & \\
\hline Gobiidae & Coryphopterus thrix & PG & \\
\hline Gobiidae & Coryphopterus urospilus & PG & \\
\hline Gobiidae & Eviota epiphanes & PG, BS & \\
\hline
\end{tabular}


Table 1 (continued)

\begin{tabular}{|c|c|c|c|}
\hline \multicolumn{2}{|l|}{ Order } & \multirow[t]{2}{*}{ Sexual pattern } & \multirow[t]{2}{*}{ Mating system } \\
\hline Family & Species & & \\
\hline Gobiidae & Fusigobius neophytus & PG & MTV polygamy \\
\hline Gobiidae & Gobiodon erythrospilus & BS & SA monogamy \\
\hline Gobiidae & Gobiodon histrio & PG, BS & SA monogamy \\
\hline Gobiidae & Gobiodon micropus & BS & SA monogamy \\
\hline Gobiidae & Gobiodon oculolinealus & BS & SA monogamy \\
\hline Gobiidae & Gobiodon okinawae & PG & SA monogamy \\
\hline Gobiidae & Gobiodon quinquestrigatus & PG, BS & SA monogamy \\
\hline Gobiidae & Lythrypnus dalli & PG, BS & MTV polygamy \\
\hline Gobiidae & Lythrypnus nesiotes & PG & \\
\hline Gobiidae & Lythrypnus phorellus & PG & \\
\hline Gobiidae & Lythrypnus pulchellus & BS & \\
\hline Gobiidae & Lythrypnus spilus & PG & \\
\hline Gobiidae & Lythrypnus zebra & BS & MTV polygamy \\
\hline Gobiidae & Paragobiodon echinocephalus & PG, BS & SA monogamy \\
\hline Gobiidae & Paragobiodon xanthosomus & PG & SA monogamy \\
\hline Gobiidae & Priolepis akihitoi & BS & \\
\hline Gobiidae & Priolepis borea & BS & \\
\hline Gobiidae & Priolepis cincta & BS & SA monogamy \\
\hline Gobiidae & Priolepis eugenius & $\mathrm{PG}, \mathrm{BS}$ & \\
\hline Gobiidae & Priolepis fallacincta & BS & \\
\hline Gobiidae & Priolepis hipoliti & PG, BS & \\
\hline Gobiidae & Priolepis inhaca & BS & \\
\hline Gobiidae & Priolepis latifascima & BS & \\
\hline Gobiidae & Priolepis semidoliata & BS & SA monogamy \\
\hline Gobiidae & Rhinogobiops nicholsii & PG & \\
\hline Gobiidae & Trimma annosum & BS & \\
\hline Gobiidae & Trimma benjamini & BS & \\
\hline Gobiidae & Trimma caesiura & BS & \\
\hline Gobiidae & Trimma cana & BS & \\
\hline Gobiidae & Trimma caudomaculatum & BS & MTV polygamy \\
\hline Gobiidae & Trimma emeryi & BS & \\
\hline Gobiidae & Trimma fangi & BS & \\
\hline Gobiidae & Trimma flammeum & BS & \\
\hline Gobiidae & Trimma flavatram & BS & \\
\hline Gobiidae & Trimma fucatum & BS & \\
\hline Gobiidae & Trimma gigantum & BS & \\
\hline Gobiidae & Trimma grammistes & BS & Harem \\
\hline Gobiidae & Trimma kudoi & BS & \\
\hline Gobiidae & Trimma lantana & BS & \\
\hline Gobiidae & Trimma macrophthalma & BS & \\
\hline Gobiidae & Trimma maiandros & BS & \\
\hline Gobiidae & Trimma marinae & BS & \\
\hline Gobiidae & Trimma milta & BS & \\
\hline Gobiidae & Trimma nasa & BS & \\
\hline Gobiidae & Trimma naudei & BS & \\
\hline Gobiidae & Trimma nесоріпит & BS & \\
\hline Gobiidae & Trimma okinawae & PG, BS & Harem \\
\hline Gobiidae & Trimma preclarum & BS & \\
\hline Gobiidae & Trimma rubromaculatum & BS & \\
\hline Gobiidae & Trimma sheppardi & BS & \\
\hline
\end{tabular}


Table 1 (continued)

\begin{tabular}{|c|c|c|c|}
\hline \multicolumn{2}{|l|}{ Order } & \multirow[t]{2}{*}{ Sexual pattern } & \multirow[t]{2}{*}{ Mating system } \\
\hline Family & Species & & \\
\hline Gobiidae & Trimma stobbsi & BS & \\
\hline Gobiidae & Trimma striatum & BS & \\
\hline Gobiidae & Trimma tauroculum & $\mathrm{BS}$ & \\
\hline Gobiidae & Trimma taylori & $\mathrm{BS}$ & \\
\hline Gobiidae & Trimma unisquamis & BS & \\
\hline Gobiidae & Trimma yanagitai & BS & \\
\hline \multicolumn{4}{|c|}{ Uncertain in ovalentaria } \\
\hline Pomacentridae & Amphiprion akallopisos & PA & NSA monogamy \\
\hline Pomacentridae & Amphiprion bicinctus & $\mathrm{PA}$ & NSA monogamy \\
\hline Pomacentridae & Amphiprion clarkii & PA & NSA monogamy \\
\hline Pomacentridae & Amphiprion frenatus & $\mathrm{PA}$ & NSA monogamy \\
\hline Pomacentridae & Amphiprion melanopus & $\mathrm{PA}$ & NSA monogamy \\
\hline Pomacentridae & Amphiprion ocellaris & PA & NSA monogamy \\
\hline Pomacentridae & Amphiprion percula & PA & NSA monogamy \\
\hline Pomacentridae & Amphiprion perideraion & $\mathrm{PA}$ & NSA monogamy \\
\hline Pomacentridae & Amphiprion polymnиs & $\mathrm{PA}$ & NSA monogamy \\
\hline Pomacentridae & Amphiprion sandaracinos & PA & NSA monogamy \\
\hline Pomacentridae & Dascyllus aruanus & PG, BS, G & Harem, MTV polygamy \\
\hline Pomacentridae & Dascyllus carneus & PG & Harem, MTV polygamy \\
\hline Pomacentridae & Dascyllus flavicaudus & $\mathrm{PG}, \mathrm{G}$ & Harem, MTV polygamy \\
\hline Pomacentridae & Dascyllus marginatus & PG & Harem, MTV polygamy \\
\hline Pomacentridae & Dascyllus melanurus & PG, G & Harem, MTV polygamy \\
\hline Pomacentridae & Dascyllus reticulatus & $\mathrm{PG}, \mathrm{G}$ & Harem, MTV polygamy \\
\hline Pseudochromidae & Anisochromis straussi & PG & \\
\hline Pseudochromidae & Ogilbyina queenslandiae & PG & \\
\hline Pseudochromidae & Pseudochromis flavivertex & $\mathrm{BS}$ & \\
\hline Pseudochromidae & Pseudochromis aldabraensis & BS & \\
\hline Pseudochromidae & Pseudochromis cyanotaenia & BS & \\
\hline Pseudochromidae & Pictichromis porphyrea & $\mathrm{BS}$ & \\
\hline \multicolumn{4}{|l|}{ Cichliformes } \\
\hline Cichlidae & Satanoperca jurupari & SH & \\
\hline Cichlidae & Metriaclima $\mathrm{cf}$. livingstoni & PG & MTV polygamy \\
\hline \multicolumn{4}{|l|}{ Cyprinodontiformes } \\
\hline Rivulidae & Kryptolebias hermaphroditus & $\mathrm{SH}, \mathrm{G}$ & \\
\hline Rivulidae & Kryptolebias marmoratus & $\mathrm{SH}, \mathrm{G}$ & \\
\hline Rivulidae & Kryptolebias ocellatus & $\mathrm{SH}, \mathrm{G}$ & \\
\hline Poeciliidae & Xiphophorus helleri & $\mathrm{PG}, \mathrm{G}$ & \\
\hline \multicolumn{4}{|l|}{ Synbranchiformes } \\
\hline Synbranchidae & Monopterus albus & PG & MTV polygamy \\
\hline Synbranchidae & Monopterus boueti & PG & \\
\hline Synbranchidae & Ophisternon bengalense & PG & \\
\hline Synbranchidae & Synbranchus marmoratus & PG & \\
\hline \multicolumn{4}{|l|}{ Trachiniformes } \\
\hline Pinguipedidae & Parapercis clathrata & PG & \\
\hline Pinguipedidae & Parapercis colias & PG & \\
\hline Pinguipedidae & Parapercis cylindrica & PG & Harem \\
\hline Pinguipedidae & Parapercis hexophtalma & PG & Harem \\
\hline Pinguipedidae & Parapercis nebulosa & PG & \\
\hline Pinguipedidae & Parapercis snyderi & PG & Harem \\
\hline Pinguipedidae & Parapercis xanthozona & PG & \\
\hline
\end{tabular}


Table 1 (continued)

\begin{tabular}{|c|c|c|c|}
\hline \multicolumn{2}{|l|}{ Order } & \multirow[t]{2}{*}{ Sexual pattern } & \multirow[t]{2}{*}{ Mating system } \\
\hline Family & Species & & \\
\hline Trichonotidae & Trichonotus filamentosus & PG & \\
\hline Creediidae & Crystallodytes cookei & $\mathrm{PA}$ & \\
\hline Creediidae & Limnichthys fasciatus & $\mathrm{PA}$ & \\
\hline Creediidae & Limnichthys nitidus & PA & \\
\hline \multicolumn{4}{|l|}{ Labriformes } \\
\hline Labridae & Achoerodus gouldii & PG & \\
\hline Labridae & Achoerodus viridis & PG & \\
\hline Labridae & Anampses geographicus & PG & \\
\hline Labridae & Bodianus axillaris & PG & \\
\hline Labridae & Bodianus diplotaenia & PG & MTV polygamy \\
\hline Labridae & Bodianus eclancheri & PG & GSP \\
\hline Labridae & Bodianus frenchii & PG & \\
\hline Labridae & Bodianus mesothorax & PG & SPA \\
\hline Labridae & Bodianus rufus & PG & Harem \\
\hline Labridae & Cheilinus chlorurus & PG & \\
\hline Labridae & Cheilinus diagrammus & PG & \\
\hline Labridae & Cheilinus fasciatus & PG & Harem \\
\hline Labridae & Cheilinus trilobatus & PG & Harem, MTV polygamy \\
\hline Labridae & Cheilinus undulatus & PG & MTV polygamy \\
\hline Labridae & Choerodon azurio & PG & \\
\hline Labridae & Choerodon cauteroma & PG & \\
\hline Labridae & Choerodon cyanodus & PG & \\
\hline Labridae & Choerodon fasciatus & PG & \\
\hline Labridae & Choerodon graphicus & PG & \\
\hline Labridae & Choerodon rubescens & PG & \\
\hline Labridae & Choerodon schoenleinii & PG & \\
\hline Labridae & Choerodon venustus & PG & \\
\hline Labridae & Cirrhilabrus temmincki & PG & MTV polygamy \\
\hline Labridae & Clepticus parrae & PG & SPA \\
\hline Labridae & Coris auricularis & PG & \\
\hline Labridae & Coris dorsomacula & PG & Harem \\
\hline Labridae & Coris gaimard & PG & MTV polygamy \\
\hline Labridae & Coris julis & PG & MTV polygamy \\
\hline Labridae & Coris variegata & PG & \\
\hline Labridae & Decodon melasma & PG & \\
\hline Labridae & Epibulus insidiator & PG & Harem, MTV polygamy \\
\hline Labridae & Gomphosus varius & PG & MTV polygamy \\
\hline Labridae & Halichoeres bivittatus & PG & MTV polygamy, GSP \\
\hline Labridae & Halichoeres garnoti & PG & MTV polygamy \\
\hline Labridae & Halichoeres maculipinna & PG & MTV polygamy \\
\hline Labridae & Halichoeres margaritaceus & PG & Harem \\
\hline Labridae & Halichoeres marginatus & PG & MTV polygamy, GSP \\
\hline Labridae & Halichoeres melanochir & PG & MTV polygamy \\
\hline Labridae & Halichoeres melanurus & PG & MTV polygamy \\
\hline Labridae & Halichoeres miniatus & PG & Harem \\
\hline Labridae & Halichoeres nebulosus & PG & \\
\hline Labridae & Halichoeres pictus & PG & \\
\hline Labridae & Halichoeres poeyi & PG & \\
\hline Labridae & Halichoeres radiatus & PG & \\
\hline Labridae & Halichoeres scapularis & PG & \\
\hline
\end{tabular}


Table 1 (continued)

\begin{tabular}{|c|c|c|c|}
\hline \multicolumn{2}{|l|}{ Order } & \multirow[t]{2}{*}{ Sexual pattern } & \multirow[t]{2}{*}{ Mating system } \\
\hline Family & Species & & \\
\hline Labridae & Halichoeres semicinctus & PG & MTV polygamy, GSP \\
\hline Labridae & Halichoeres tenuispinnis & PG & MTV polygamy \\
\hline Labridae & Halichoeres trimaculatus & PG, BS & MTV polygamy, GSP \\
\hline Labridae & Hemigymnus melapterus & PG & \\
\hline Labridae & Hologymnosus annulatus & PG & \\
\hline Labridae & Iniistius dea & PG & \\
\hline Labridae & Iniistius geisha & PG & \\
\hline Labridae & Iniistius pentadactylus & PG & Harem \\
\hline Labridae & Labrichthys unilineatus & PG & Harem \\
\hline Labridae & Labroides dimidiatus & PG, BS & Harem \\
\hline Labridae & Labrus bergylta & PG & \\
\hline Labridae & Labrus merula & PG & \\
\hline Labridae & Labrus mixtus & PG & \\
\hline Labridae & Labrus viridis & PG & \\
\hline Labridae & Lachnolaimus maximus & PG & Harem \\
\hline Labridae & Macropharyngodon moyeri & PG & Harem \\
\hline Labridae & Notolabrus celidotus & PG & MTV polygamy \\
\hline Labridae & Notolabrus gymnogenis & PG & \\
\hline Labridae & Notolabrus parilus & PG & \\
\hline Labridae & Notolabrus tetricus & PG & MTV polygamy \\
\hline Labridae & Ophthalmolepis lineolatus & PG & \\
\hline Labridae & Parajulis poecilepterus & PG & GSP \\
\hline Labridae & Pictilabrus laticlavius & PG & MTV polygamy \\
\hline Labridae & Pseudocheilinops ataenia & PG & \\
\hline Labridae & Pseudocheilinus evanidus & PG & \\
\hline Labridae & Pseudocheilinus hexataenia & PG & Harem \\
\hline Labridae & Pseudolabrus guentheri & PG & \\
\hline Labridae & Pseudolabrus rubicundus & PG & MTV polygamy \\
\hline Labridae & Pseudolabrus sieboldi & PG & MTV polygamy \\
\hline Labridae & Pteragogus aurigarius & PG & MTV polygamy \\
\hline Labridae & Semicossyphus darwini & PG & \\
\hline Labridae & Semicossyphus pulcher & PG & MTV polygamy \\
\hline Labridae & Semicossyphus reticulatus & PG & \\
\hline Labridae & Stethojulis balteata & PG & \\
\hline Labridae & Stethojulis interrupta & PG & MTV polygamy \\
\hline Labridae & Stethojulis strigiventer & PG & \\
\hline Labridae & Stethojulis trilineata & PG & MTV polygamy \\
\hline Labridae & Suezichthys ornatus & PG & MTV polygamy \\
\hline Labridae & Symphodus melanocercus & PG & MTV polygamy \\
\hline Labridae & Symphodus tinca & $\mathrm{PG}, \mathrm{G}$ & MTV polygamy \\
\hline Labridae & Thalassoma bifasciatum & PG & MTV polygamy, GSP \\
\hline Labridae & Thalassoma cupido & PG & MTV polygamy, GSP \\
\hline Labridae & Thalassoma duperrey & PG & MTV polygamy, GSP \\
\hline Labridae & Thalassoma hardwicke & PG & MTV polygamy, GSP \\
\hline Labridae & Thalassoma jansenii & PG & MTV polygamy, GSP \\
\hline Labridae & Thalassoma lucasanum & PG & MTV polygamy, GSP \\
\hline Labridae & Thalassoma lunare & PG & MTV polygamy, GSP \\
\hline Labridae & Thalassoma lutescens & PG & MTV polygamy, GSP \\
\hline Labridae & Thalassoma pavo & PG & MTV polygamy, GSP \\
\hline Labridae & Thalassoma purpureum & PG & \\
\hline
\end{tabular}


Table 1 (continued)

\begin{tabular}{|c|c|c|c|}
\hline \multicolumn{2}{|l|}{ Order } & \multirow[t]{2}{*}{ Sexual pattern } & \multirow[t]{2}{*}{ Mating system } \\
\hline Family & Species & & \\
\hline Labridae & Thalassoma quinquevittatum & PG & MTV polygamy, GSP \\
\hline Labridae & Xyrichtys martinicensis & PG & Harem \\
\hline Labridae & Xyrichtys novacula & PG & Harem \\
\hline Odacidae & Odax pullus & PG & \\
\hline Scaridae & Calotomus carolinus & PG & MTV polygamy \\
\hline Scaridae & Calotomus japonicus & PG & MTV polygamy \\
\hline Scaridae & Calotomus spinidens & PG & MTV polygamy \\
\hline Scaridae & Cetoscarus bicolor & PG & \\
\hline Scaridae & Chlorurus sordidus & PG & MTV polygamy \\
\hline Scaridae & Chlorurus spilurus & PG & \\
\hline Scaridae & Cryptotomus roseus & PG & MTV polygamy \\
\hline Scaridae & Hipposcarus harid & PG & \\
\hline Scaridae & Scarus ferrugineus & PG & \\
\hline Scaridae & Scarus festivus & PG & \\
\hline Scaridae & Scarus forsteni & PG & MTV polygamy \\
\hline Scaridae & Scarus frenatus & PG & Harem \\
\hline Scaridae & Scarus ghobban & PG & \\
\hline Scaridae & Scarus globiceps & PG & MTV polygamy, GSP \\
\hline Scaridae & Scarus iseri & PG & Harem, GSP \\
\hline Scaridae & Scarus niger & PG & MTV polygamy, GSP \\
\hline Scaridae & Scarus oviceps & PG & MTV polygamy \\
\hline Scaridae & Scarus psittacus & PG & MTV polygamy \\
\hline Scaridae & Scarus rivulatus & PG & MTV polygamy \\
\hline Scaridae & Scarus rubroviolaceus & PG & \\
\hline Scaridae & Scarus russelii & PG & \\
\hline Scaridae & Scarus scaber & PG & \\
\hline Scaridae & Scarus schlegeli & PG & MTV polygamy \\
\hline Scaridae & Scarus spinus & PG & \\
\hline Scaridae & Scarus taeniopterus & PG & \\
\hline Scaridae & Scarus tricolor & PG & \\
\hline Scaridae & Scarus vetula & PG & Harem, MTV polygamy \\
\hline Scaridae & Scarus viridifucatus & PG & \\
\hline Scaridae & Sparisoma atomarium & PG & Harem \\
\hline Scaridae & Sparisoma aurofrenatum & PG & Harem \\
\hline Scaridae & Sparisoma chrysopterum & PG & MTV polygamy \\
\hline Scaridae & Sparisoma cretense & PG, G & Harem \\
\hline Scaridae & Sparisoma radians & PG & Harem, MTV polygamy, GSP \\
\hline Scaridae & Sparisoma rubripinne & PG & MTV polygamy, GSP \\
\hline Scaridae & Sparisoma viride & PG & MTV polygamy \\
\hline \multicolumn{4}{|l|}{ Perciformes } \\
\hline Centropomidae & Centropomus undecimalis & PA & \\
\hline Latidae & Lates calcarifer & PA & \\
\hline Polynemidae & Galeoides decadactylus & $\mathrm{PA}, \mathrm{G}$ & \\
\hline Polynemidae & Filimanus heptadactyla & $\mathrm{SH}, \mathrm{G}$ & \\
\hline Polynemidae & Polydactylus macrochir & PA & \\
\hline Polynemidae & Polydactylus microstomus & $\mathrm{SH}, \mathrm{G}$ & \\
\hline Polynemidae & Polydactylus quadrifilis & PA & \\
\hline Terapontidae & Bidyanus bidyanus & PA & \\
\hline Terapontidae & Mesopristes cancellatus & PA & \\
\hline Serranidae (Epinephelinae) & Cephalopholis argus & PG & Harem \\
\hline
\end{tabular}


Table 1 (continued)

\begin{tabular}{|c|c|c|c|}
\hline \multicolumn{2}{|l|}{ Order } & \multirow[t]{2}{*}{ Sexual pattern } & \multirow[t]{2}{*}{ Mating system } \\
\hline Family & Species & & \\
\hline Serranidae (Epinephelinae) & Cephalopholis boenak & PG, BS & \\
\hline Serranidae (Epinephelinae) & Cephalopholis cruentata & PG & Harem \\
\hline Serranidae (Epinephelinae) & Cephalopholis cyanostigma & PG & Harem \\
\hline Serranidae (Epinephelinae) & Cephalopholis fulva & PG & Harem \\
\hline Serranidae (Epinephelinae) & Cephalopholis hemistiktos & PG & Harem \\
\hline Serranidae (Epinephelinae) & Cephalopholis miniata & PG & Harem \\
\hline Serranidae (Epinephelinae) & Cephalopholis panamensis & PG & Harem \\
\hline Serranidae (Epinephelinae) & Cephalopholis taeniops & PG & \\
\hline Serranidae (Epinephelinae) & Cephalopholis urodeta & PG & \\
\hline Serranidae (Epinephelinae) & Epinephelus adscensionis & PG & Harem \\
\hline Serranidae (Epinephelinae) & Epinephelus aeneus & PG & \\
\hline Serranidae (Epinephelinae) & Epinephelus akaara & PG, BS & \\
\hline Serranidae (Epinephelinae) & Epinephelus andersoni & PG & \\
\hline Serranidae (Epinephelinae) & Epinephelus bruneus & PG, BS & \\
\hline Serranidae (Epinephelinae) & Epinephelus chlorostigma & PG & \\
\hline Serranidae (Epinephelinae) & Epinephelus coioides & PG & \\
\hline Serranidae (Epinephelinae) & Epinephelus diacanthus & PG & \\
\hline Serranidae (Epinephelinae) & Epinephelus drummondhayi & PG & \\
\hline Serranidae (Epinephelinae) & Epinephelus fasciatus & PG & \\
\hline Serranidae (Epinephelinae) & Epinephelus fuscoguttatus & PG & SPA \\
\hline Serranidae (Epinephelinae) & Epinephelus guttatus & PG & SPA \\
\hline Serranidae (Epinephelinae) & Epinephelus itajara & $\mathrm{PG}, \mathrm{G}$ & GSP \\
\hline Serranidae (Epinephelinae) & Epinephelus labriformis & PG & \\
\hline Serranidae (Epinephelinae) & Epinephelus malabaricus & PG & \\
\hline Serranidae (Epinephelinae) & Epinephelus marginatus & PG & MTV polygamy \\
\hline Serranidae (Epinephelinae) & Epinephelus merra & PG & SPA \\
\hline Serranidae (Epinephelinae) & Epinephelus morio & PG & \\
\hline Serranidae (Epinephelinae) & Epinephelus ongus & PG & SPA \\
\hline Serranidae (Epinephelinae) & Epinephelus rivulatus & PG & \\
\hline Serranidae (Epinephelinae) & Epinephelus striatus & $\mathrm{PG}, \mathrm{G}$ & GSP \\
\hline Serranidae (Epinephelinae) & Epinephelus tauvina & PG & \\
\hline Serranidae (Epinephelinae) & Hyporthodus flavolimbatus & PG & \\
\hline Serranidae (Epinephelinae) & Hyporthodus niveatus & PG & \\
\hline Serranidae (Epinephelinae) & Hyporthodus quernus & PG & \\
\hline Serranidae (Epinephelinae) & Mycteroperca bonaci & PG & \\
\hline Serranidae (Epinephelinae) & Mycteroperca interstitialis & PG & \\
\hline Serranidae (Epinephelinae) & Mycteroperca microlepis & PG & SPA \\
\hline Serranidae (Epinephelinae) & Mycteroperca olfax & PG & SPA \\
\hline Serranidae (Epinephelinae) & Mycteroperca phenax & PG & SPA \\
\hline Serranidae (Epinephelinae) & Mycteroperca rubra & PG & SPA \\
\hline Serranidae (Epinephelinae) & Mycteroperca venenosa & PG & GSP \\
\hline Serranidae (Epinephelinae) & Plectropomus laevis & PG & \\
\hline Serranidae (Epinephelinae) & Plectropoтus leopardus & PG & SPA \\
\hline Serranidae (Epinephelinae) & Plectropoтиs maculatus & PG & \\
\hline Serranidae (Serraninae) & Bullisichthys caribbaeus & SH & \\
\hline Serranidae (Serraninae) & Centropristis striata & PG & \\
\hline Serranidae (Serraninae) & Centropristis ocyurus & PG & \\
\hline Serranidae (Serraninae) & Chelidoperca hirundinacea & PG & \\
\hline Serranidae (Serraninae) & Diplectrum bivittatum & SH & \\
\hline Serranidae (Serraninae) & Diplectrum formosum & SH & \\
\hline
\end{tabular}


Table 1 (continued)

\begin{tabular}{|c|c|c|c|}
\hline \multicolumn{2}{|l|}{ Order } & \multirow[t]{2}{*}{ Sexual pattern } & \multirow[t]{2}{*}{ Mating system } \\
\hline Family & Species & & \\
\hline Serranidae (Serraninae) & Diplectrum macropoma & SH & \\
\hline Serranidae (Serraninae) & Diplectrum pacificum & SH & \\
\hline Serranidae (Serraninae) & Diplectrum rostrum & SH & \\
\hline Serranidae (Serraninae) & Hypoplectrus aberrans & SH & SA monogamy \\
\hline Serranidae (Serraninae) & Hypoplectrus chlorurus & $\mathrm{SH}$ & SA monogamy \\
\hline Serranidae (Serraninae) & Hypoplectrus nigricans & $\mathrm{SH}$ & SA monogamy \\
\hline Serranidae (Serraninae) & Hypoplectrus puella & SH & SA monogamy \\
\hline Serranidae (Serraninae) & Hypoplectrus unicolor & SH & SA monogamy \\
\hline Serranidae (Serraninae) & Paralabrax maculatofasciatus & $\mathrm{PG}, \mathrm{G}$ & \\
\hline Serranidae (Serraninae) & Serraniculus pumilio & SH & \\
\hline Serranidae (Serraninae) & Serranus annularis & SH & \\
\hline Serranidae (Serraninae) & Serranus atricauda & SH & \\
\hline Serranidae (Serraninae) & Serranus auriga & SH & \\
\hline Serranidae (Serraninae) & Serranus baldwini & SH & Harem \\
\hline Serranidae (Serraninae) & Serranus cabrilla & SH & \\
\hline Serranidae (Serraninae) & Serranus hepatus & SH & \\
\hline Serranidae (Serraninae) & Serranus phoebe & SH & \\
\hline Serranidae (Serraninae) & Serranus psittacinus & SH & Harem \\
\hline Serranidae (Serraninae) & Serranus scriba & SH & \\
\hline Serranidae (Serraninae) & Serranus subligarius & SH & SA monogamy \\
\hline Serranidae (Serraninae) & Serranus tabacarius & SH & SA monogamy \\
\hline Serranidae (Serraninae) & Serranus tigrinus & SH & SA monogamy \\
\hline Serranidae (Serraninae) & Serranus tortugarum & $\mathrm{SH}$ & SA monogamy \\
\hline Serranidae (Grammistini) & Pseudogramma gregoryi & $\mathrm{SH}$ & \\
\hline Serranidae (Grammistini) & Rypticus saponaceus & PG & \\
\hline Serranidae (Grammistini) & Rypticus subbifrenatus & PG & \\
\hline Serranidae (Anthiinae) & Anthias anthias & PG & \\
\hline Serranidae (Anthiinae) & Anthias nicholsi & PG & \\
\hline Serranidae (Anthiinae) & Anthias noeli & PG & \\
\hline Serranidae (Anthiinae) & Baldwinella vivanus & PG & \\
\hline Serranidae (Anthiinae) & Hemanthias leptus & PG & \\
\hline Serranidae (Anthiinae) & Hemanthias peruanus & PG & \\
\hline Serranidae (Anthiinae) & Hypoplectrodes huntii & PG & \\
\hline Serranidae (Anthiinae) & Hypoplectrodes maccullochi & PG & \\
\hline Serranidae (Anthiinae) & Pronotogrammus martinicensis & PG & \\
\hline Serranidae (Anthiinae) & Pseudanthias conspicuus & PG & \\
\hline Serranidae (Anthiinae) & Pseudanthias elongatus & PG & \\
\hline Serranidae (Anthiinae) & Pseudanthias pleurotaenia & PG & \\
\hline Serranidae (Anthiinae) & Pseudanthias rubrizonatus & PG & \\
\hline Serranidae (Anthiinae) & Pseudanthias squamipinnis & PG & MTV polygamy \\
\hline Serranidae (Anthiinae) & Sacura margaritacea & PG & \\
\hline Pomacanthidae & Centropyge acanthops & PG, BS & \\
\hline Pomacanthidae & Centropyge bicolor & PG & Harem \\
\hline Pomacanthidae & Centropyge ferrugata & PG, BS & Harem \\
\hline Pomacanthidae & Centropyge fisheri & PG, BS & \\
\hline Pomacanthidae & Centropyge flavissimus & BS & Harem \\
\hline Pomacanthidae & Centropyge heraldi & PG & Harem \\
\hline Pomacanthidae & Centropyge interruptus & PG & Harem \\
\hline Pomacanthidae & Centropyge multispinus & PG & \\
\hline Pomacanthidae & Centropyge potteri & PG & Harem \\
\hline
\end{tabular}


Table 1 (continued)

\begin{tabular}{|c|c|c|c|}
\hline \multicolumn{2}{|l|}{ Order } & \multirow[t]{2}{*}{ Sexual pattern } & \multirow[t]{2}{*}{ Mating system } \\
\hline Family & Species & & \\
\hline Pomacanthidae & Centropyge tibicen & PG & Harem \\
\hline Pomacanthidae & Centropyge vrolicki & PG & Harem \\
\hline Pomacanthidae & Chaetodontoplus septentrionalis & PG & Harem \\
\hline Pomacanthidae & Genicanthus bellus & PG & \\
\hline Pomacanthidae & Genicanthus caudovittatus & PG & Harem \\
\hline Pomacanthidae & Genicanthus lamarck & PG & Harem \\
\hline Pomacanthidae & Genicanthus melanospilos & PG & Harem \\
\hline Pomacanthidae & Genicanthus personatus & PG & Harem \\
\hline Pomacanthidae & Genicanthus semifasciatus & PG & Harem \\
\hline Pomacanthidae & Genicanthus watanabei & PG & \\
\hline Pomacanthidae & Holacanthus passer & $\mathrm{PG}, \mathrm{G}$ & Harem \\
\hline Pomacanthidae & Holacanthus tricolor & PG & Harem \\
\hline Pomacanthidae & Pomacanthus zonipectus & PG & \\
\hline Pomacanthidae & Apolemichthys trimaculatus & PG & \\
\hline Malacanthidae & Malacanthus plumieri & PG & Harem \\
\hline Cirrhitidae & Amblycirrhitus pinos & PG & \\
\hline Cirrhitidae & Cirrhitichthys aprinus & PG & Harem \\
\hline Cirrhitidae & Cirrhitichthys aureus & PG, BS & \\
\hline Cirrhitidae & Cirrhitichthys falco & PG, BS & Harem \\
\hline Cirrhitidae & Cirrhitichthys oxycephalus & PG & Harem \\
\hline Cirrhitidae & Neocirrhites armatus & PG & Harem \\
\hline Eleginopsidae & Eleginops maclovinus & PA & \\
\hline \multicolumn{4}{|l|}{ Scorpaeniformes } \\
\hline Platycephalidae & Cociella crocodila & PA & \\
\hline Platycephalidae & Inegocia japonica & PA & Random mating \\
\hline Platycephalidae & Kumococius rodericensis & $\mathrm{PA}$ & \\
\hline Platycephalidae & Onigocia macrolepis & PA & \\
\hline Platycephalidae & Platycephalus sp. & $\mathrm{PA}$ & Random mating \\
\hline Platycephalidae & Suggrundus meerdervoorti & PA & \\
\hline Platycephalidae & Thysanophrys celebica & $\mathrm{PA}$ & Random mating \\
\hline Scorpaenidae & Caracanthus unipinna & PG & Harem \\
\hline \multicolumn{4}{|l|}{ Moroniformes } \\
\hline Moronidae & Morone saxatilis & PA & \\
\hline \multicolumn{4}{|l|}{ Spariformes } \\
\hline Nemipteridae & Scolopsis monogramma & PG & \\
\hline Nemipteridae & Scolopsis taenioptera & PG & \\
\hline Lethrinidae & Lethrinus nebulosus & $\mathrm{PG}, \mathrm{G}$ & \\
\hline Lethrinidae & Lethrinus genivittatus & PG & \\
\hline Lethrinidae & Lethrinus lentjan & PG & \\
\hline Lethrinidae & Lethrinus variegatus & PG & \\
\hline Lethrinidae & Lethrinus miniatus & PG & \\
\hline Lethrinidae & Lethrinus rubrioperculatus & PG & \\
\hline Lethrinidae & Lethrinus atkinsoni & PG & \\
\hline Lethrinidae & Lethrinus harak & PG & \\
\hline Lethrinidae & Lethrinus ornatus & PG & \\
\hline Lethrinidae & Lethrinus ravus & PG & \\
\hline Sparidae & Acanthopagrus australis & $\mathrm{PA}$ & \\
\hline Sparidae & Acanthopagrus berda & PA & SPA \\
\hline Sparidae & Acanthopagrus schlegelii & PA & \\
\hline Sparidae & Acanthopagrus latus & PA & \\
\hline
\end{tabular}


Table 1 (continued)

\begin{tabular}{|c|c|c|c|}
\hline \multicolumn{2}{|l|}{ Order } & \multirow[t]{2}{*}{ Sexual pattern } & \multirow[t]{2}{*}{ Mating system } \\
\hline Family & Species & & \\
\hline Sparidae & Calamus leucosteus & PG & \\
\hline Sparidae & Chrysoblephus cristiceps & PG & \\
\hline Sparidae & Chrysoblephus puniceus & PG & \\
\hline Sparidae & Chrysoblephus laticeps & PG & \\
\hline Sparidae & Dentex gibbosus & PG & \\
\hline Sparidae & Dentex tumifrons & PG & \\
\hline Sparidae & Diplodus annularis & $\mathrm{PA}, \mathrm{G}$ & \\
\hline Sparidae & Diplodus capensis & $\mathrm{PA}, \mathrm{G}$ & \\
\hline Sparidae & Diplodus puntazzo & $\mathrm{PA}, \mathrm{G}$ & \\
\hline Sparidae & Diplodus sargus & $\mathrm{PA}, \mathrm{G}$ & \\
\hline Sparidae & Diplodus vulgaris & $\mathrm{PA}, \mathrm{G}$ & \\
\hline Sparidae & Lithognathus mormyrus & $\mathrm{PA}, \mathrm{G}$ & \\
\hline Sparidae & Pagellus acarne & PA, G & \\
\hline Sparidae & Pagellus bogaraveo & PA, G & \\
\hline Sparidae & Pagellus erythrinus & PG & \\
\hline Sparidae & Pagrus auriga & PG & \\
\hline Sparidae & Pagrus caeruleostictus & PG & \\
\hline Sparidae & Pagrus ehrenbergii & $\mathrm{PG}, \mathrm{G}$ & \\
\hline Sparidae & Pagrus major & PG, G & \\
\hline Sparidae & Pagrus pagrus & PG & \\
\hline Sparidae & Rhabdosargus sarba & $\mathrm{PA}, \mathrm{G}$ & \\
\hline Sparidae & Sarpa salpa & $\mathrm{PA}, \mathrm{G}$ & SPA \\
\hline Sparidae & Sparidentex hasta & PA & \\
\hline Sparidae & Sparus aurata & PA & GSP \\
\hline Sparidae & Spicara chryselis & PG & \\
\hline Sparidae & Spicara smaris & PG & \\
\hline Sparidae & Spicara maena & PG & \\
\hline Sparidae & Spondyliosoma cantharus & PG & \\
\hline \multicolumn{4}{|c|}{ Tetraodontiformes } \\
\hline Balistidae & Sufflamen chrysopterus & PG & Harem \\
\hline
\end{tabular}

$S H$ simultaneous hermaphroditism, $P A$ protandry, $P G$ protogyny, $B S$ bidirectional sex change or reversed sex change in protogynous species, $G$ gonochorism, SA monogamy size-assortative monogamy, NSA monogamy non-size-assortative monogamy, Harem harem polygyny, MTV polygamy male-territory-visiting polygamy, GSP group spawning, SPA spawning aggregation unknown detailed mating system, blank unknown. Facultative monogamy in polygamous species is not shown. If intraspecific variation has been reported in sexual pattern or mating system, two or more types are shown. Detailed data of each species and references are given in Table S1 and S2, respectively

Serranidae, Sparidae, Labridae, and Gobiidae, respectively (26, 16, 98, and 50 spp., respectively). Most cases of BS in Gobiidae have been determined by gonad histology or aquarium experiments (e.g., Sunobe et al. 2017; Table S1), and the frequency of each sex change direction in the field is known only in a few species (Kuwamura et al. 1994; Manabe et al. 2007).

Three types of hermaphroditism were found in Pomacentridae (PA, PG, and BS) and Serranidae (SH, PG, and BS) (Table 2), while two types were found in Muraenidae and Polynemidae (SH and PA), Cichlidae (SH and PG), Sparidae (PA and PG), and Gobiidae, Pseudochromidae, Labridae, Pomacanthidae, and Cirrhitidae (PG and BS). This indicated that different types of hermaphroditism have appeared in different lineages repeatedly (see Mank et al. 2006), and the sexual pattern is often highly variable in expression within a single family.

The frequency of hermaphroditic fish families in each order is presented in Table 3. We found hermaphrodites in 17 orders (27\% of 63 orders of Teleostei). Two families in Series Ovalentaria, Pomacentridae and Pseudochromidae, are not classified into orders by Nelson et al. (2016), but were counted as belonging to the same order here, for convenience. Among 17 orders, PG was the most abundant (20 families, $49 \%$ of 41 hermaphroditic families), BS the least abundant (7 families, 17\%), and SH and PA had similar abundances (13 and 14 families, 32\% and 34\%, respectively). The tendency was somewhat different from that of 
Table 2 Frequency of hermaphroditic fish species in each family. Order and family names are arranged following Nelson et al. (2016). Summed up from Table 1

\begin{tabular}{|c|c|c|c|c|c|c|c|c|c|}
\hline \multirow[t]{2}{*}{ Order } & \multirow[t]{2}{*}{ Family } & \multicolumn{6}{|c|}{$\begin{array}{l}\text { Number of hermaphroditic spe- } \\
\text { cies }\end{array}$} & \multirow{2}{*}{$\begin{array}{l}\text { Total } \\
\text { number of } \\
\text { species }\end{array}$} & \multirow[t]{2}{*}{ Habitat** } \\
\hline & & $\mathrm{SH}$ & PA & PG & BS & Total & $\% *$ & & \\
\hline Anguilliformes & Muraenidae & 3 & 1 & 0 & 0 & 4 & 2.0 & 200 & M \\
\hline Clupeiformes & Clupeidae & 0 & 2 & 0 & 0 & 2 & 0.9 & 218 & M \\
\hline Cypriniformes & Cobitidae & 0 & 1 & 0 & 0 & 1 & 0.5 & 195 & $\mathrm{~F}$ \\
\hline Stomiiformes & Gonostomatidae & 0 & 5 & 0 & 0 & 5 & 16.1 & 31 & M \\
\hline \multirow[t]{8}{*}{ Aulopiformes } & Ipnopidae & 9 & 0 & 0 & 0 & 9 & 28.1 & 32 & M \\
\hline & Giganturidae & 2 & 0 & 0 & 0 & 2 & 100.0 & 2 & M \\
\hline & Bathysauridae & 1 & 0 & 0 & 0 & 1 & 50.0 & 2 & M \\
\hline & Chlorophthalmidae & 3 & 0 & 0 & 0 & 3 & 17.6 & 17 & M \\
\hline & Notosudidae & 1 & 0 & 0 & 0 & 1 & 5.9 & 17 & M \\
\hline & Scopelarchidae & 2 & 0 & 0 & 0 & 2 & 11.1 & 18 & M \\
\hline & Paralepididae & 1 & 0 & 0 & 0 & 1 & 3.7 & 27 & M \\
\hline & Alepisauridae & 1 & 0 & 0 & 0 & 1 & 11.1 & 9 & M \\
\hline Gobiiformes & Gobiidae & 0 & 0 & 24 & 50 & 66 & 4.9 & 1359 & $\mathrm{M}(\& \mathrm{~F})$ \\
\hline \multirow[t]{2}{*}{ Uncertain in ovalentaria } & Pomacentridae & 0 & 10 & 6 & 1 & 16 & 4.1 & 387 & M \\
\hline & Pseudochromidae & 0 & 0 & 2 & 4 & 6 & 3.9 & 152 & M \\
\hline Cichliformes & Cichlidae & 1 & 0 & 1 & 0 & 2 & 0.1 & 1762 & $\mathrm{~F}$ \\
\hline \multirow[t]{2}{*}{ Cyprinodontiformes } & Rivulidae & 3 & 0 & 0 & 0 & 3 & 0.8 & 370 & $\mathrm{~F}$ \\
\hline & Poeciliidae & 0 & 0 & 1 & 0 & 1 & 0.3 & 353 & $\mathrm{~F}$ \\
\hline Synbranchiformes & Synbranchidae & 0 & 0 & 4 & 0 & 4 & 17.4 & 23 & $\mathrm{~F}$ \\
\hline \multirow[t]{3}{*}{ Trachiniformes } & Pinguipedidae & 0 & 0 & 7 & 0 & 7 & 8.5 & 82 & M \\
\hline & Trichonotidae & 0 & 0 & 1 & 0 & 1 & 10.0 & 10 & M \\
\hline & Creediidae & 0 & 3 & 0 & 0 & 3 & 16.7 & 18 & M \\
\hline \multirow[t]{3}{*}{ Labriiformes } & Labridae & 0 & 0 & 98 & 2 & 98 & 18.9 & 519 & M \\
\hline & Odacidae & 0 & 0 & 1 & 0 & 1 & 8.3 & 12 & M \\
\hline & Scaridae & 0 & 0 & 35 & 0 & 35 & 35.4 & 99 & M \\
\hline \multirow[t]{9}{*}{ Perciformes } & Centropomidae & 0 & 1 & 0 & 0 & 1 & 8.3 & 12 & $\mathrm{M} \& \mathrm{~F}$ \\
\hline & Latidae & 0 & 1 & 0 & 0 & 1 & 7.7 & 13 & $\mathrm{M} \& \mathrm{~F}$ \\
\hline & Polynemidae & 2 & 3 & 0 & 0 & 5 & 11.9 & 42 & M \\
\hline & Terapontidae & 0 & 2 & 0 & 0 & 2 & 3.8 & 52 & $(\mathrm{M} \&) \mathrm{F}$ \\
\hline & Serranidae & 26 & 0 & 66 & 3 & 92 & 17.1 & 538 & M \\
\hline & Pomacanthidae & 0 & 0 & 22 & 4 & 23 & 25.8 & 89 & M \\
\hline & Malacanthidae & 0 & 0 & 1 & 0 & 1 & 2.2 & 45 & M \\
\hline & Cirrhitidae & 0 & 0 & 6 & 2 & 6 & 18.2 & 33 & M \\
\hline & Eleginopsidae & 0 & 1 & 0 & 0 & 1 & 100.0 & 1 & M \\
\hline \multirow[t]{2}{*}{ Scorpaeniformes } & Platycephalidae & 0 & 7 & 0 & 0 & 7 & 8.8 & 80 & M \\
\hline & Scorpaenidae & 0 & 0 & 1 & 0 & 1 & 0.2 & 454 & M \\
\hline Moroniformes & Moronidae & 0 & 1 & 0 & 0 & 1 & 25.0 & 4 & $\mathrm{M} \& \mathrm{~F}$ \\
\hline \multirow[t]{3}{*}{ Spariformes } & Nemipteridae & 0 & 0 & 2 & 0 & 2 & 3.0 & 67 & M \\
\hline & Lethrinidae & 0 & 0 & 10 & 0 & 10 & 26.3 & 38 & M \\
\hline & Sparidae & 0 & 16 & 16 & 0 & 32 & 84.2 & 38 & M \\
\hline Tetraodontiformes & Balistidae & 0 & 0 & 1 & 0 & 1 & 2.4 & 42 & M \\
\hline Total number of species & & 55 & 54 & 305 & 66 & 461 & & & \\
\hline
\end{tabular}

SH simultaneous hermaphroditism, $P A$ protandry, $P G$ protogyny, $B S$ bidirectional sex change or reversed sex change in protogynous species. When two or more types are reported within a species, we counted them in each column

*Percentage of hermaphroditic species in each family (total number of species after Nelson et al. 2016)

** $M$ marine, $F$ fresh water (after Nelson et al. 2016). In parentheses if no hermaphrodites have been reported from the habitat 
Table 3 Frequency of hermaphroditic fish families in each order. Order names are arranged following Nelson et al. (2016). Summed up from Table 2

\begin{tabular}{|c|c|c|c|c|c|c|c|c|}
\hline \multirow[t]{2}{*}{ Order } & \multicolumn{6}{|c|}{$\begin{array}{l}\text { Number of families including her- } \\
\text { maphrodites }\end{array}$} & \multirow[t]{2}{*}{$\begin{array}{l}\text { Total number } \\
\text { of families }\end{array}$} & \multirow[t]{2}{*}{ Group names in Fig. 1} \\
\hline & $\mathrm{SH}$ & PA & PG & $\mathrm{BS}$ & Total & $\% *$ & & \\
\hline Anguilliformes & 1 & 1 & 0 & 0 & 1 & 5.3 & 19 & Elopomorpha \\
\hline Clupeiformes & 0 & 1 & 0 & 0 & 1 & 20.0 & 5 & Clupeiformes \\
\hline Cypriniformes & 0 & 1 & 0 & 0 & 1 & 7.7 & 13 & Cypriniformes \\
\hline Stomiiformes & 0 & 1 & 0 & 0 & 1 & 20.0 & 5 & Stomiatiformes \\
\hline Aulopiformes & 8 & 0 & 0 & 0 & 8 & 53.3 & 15 & Aulopiformes \\
\hline Gobiiformes & 0 & 0 & 1 & 1 & 1 & 12.5 & 8 & Gobiaria \\
\hline Uncertain** in ovalentaria & 0 & 1 & 2 & 2 & 2 & 25.0 & 8 & Ovalentaria \\
\hline Cichliformes & 1 & 0 & 1 & 0 & 1 & 50.0 & 2 & Ovalentaria \\
\hline Cyprinodontiformes & 1 & 0 & 1 & 0 & 2 & 20.0 & 10 & Ovalentaria \\
\hline Synbranchiformes & 0 & 0 & 1 & 0 & 1 & 33.3 & 3 & Anabantaria \\
\hline Trachiniformes & 0 & 1 & 2 & 0 & 3 & 27.3 & 11 & Eupercaria \\
\hline Labriiformes & 0 & 0 & 3 & 1 & 3 & 100.0 & 3 & Eupercaria \\
\hline Perciformes & 2 & 5 & 4 & 3 & 9 & 14.5 & 62 & Eupercaria \\
\hline Scorpaeniformes & 0 & 1 & 1 & 0 & 2 & 4.9 & 41 & Eupercaria \\
\hline Moroniformes & 0 & 1 & 0 & 0 & 1 & 33.3 & 3 & Eupercaria \\
\hline Spariformes & 0 & 1 & 3 & 0 & 3 & 50.0 & 6 & Eupercaria \\
\hline Tetraodontiformes & 0 & 0 & 1 & 0 & 1 & 10.0 & 10 & Eupercaria \\
\hline Total number of families & 13 & 14 & 20 & 7 & 41 & & & \\
\hline
\end{tabular}

$S H$ simultaneous hermaphroditism, $P A$ protandry, $P G$ protogyny, $B S$ bidirectional sex change or reversed sex change in protogynous species. When two or more types are reported within a family, we counted them in each column

*Percentage of hermaphroditic families in each order (total number of families after Nelson et al. 2016)

**Treated as one order for convenience the number of species. The number of species with BS was higher than that with SH or PA because of the extensive surveys on BS species of Gobiidae (Table 2, S1). The percentage of hermaphroditic families in an order varied widely from $4.9 \%$ ( 2 out of 41 families in Scorpaeniformes) to $100 \%$ (all three families in Labriformes) (Table 3). All four types were only found in Perciformes, and three types (PA, PG, and BS) in the tentative order (Pomacentridae-Pseudochromidae) in Ovalentaria.

By combining our list with the phylogenetic tree of Betancur-R et al. (2017), it was indicated that SH and PA have evolved several times in not-closely related lineages; $\mathrm{SH}$ in Elopomorpha, Aulopiformes, Ovalentaria, and Eupercaria, and PA in Elopomorpha, Clupeiformes, Cypriniformes, Stomiatiformes, Ovalentaria, and Eupercaria (Fig. 1). For Elopomorpha, PG was suggested by Fishelson (1992) in nine species of Muraenidae based on gonad histology of few samples (Table S1), but functional protogynous sex change has not been confirmed (Sadovy de Mitcheson and Liu 2008). Therefore, it is suggested that PG (and BS) has evolved only in four lineages (Gobiaria, Anabantaria, Ovalentaria, and Eupercaria) of Percomorpha relatively recently (Fig. 1). The different distribution patterns in the phylogenetic tree of the former two types ( $\mathrm{SH}$ and $\mathrm{PA}$ ) and the latter (PG and $\mathrm{BS}$ ) are interesting, and may also be related to the difference in mating systems.

Habitat (marine or freshwater) and hermaphroditism. Among hermaphroditic fishes, the number of families only inhabiting freshwater ( 6 families, $15 \%$ of all hermaphroditic families) was much lower than that of marine only families (32 families, 78\%) (Table 2). All four types of hermaphroditism were found in marine families, while three types (except BS) were found in freshwater families. In freshwater families, the number of species of each type was similar ( $\mathrm{SH}, 4$; PA, 3; PG, 6), and the total number of freshwater hermaphroditic species (13 spp., 3\% of all hermaphroditic species) was much lower than that in marine families (445 spp., 97\%). The percentage of freshwater species in hermaphroditic species (3\%) was significantly lower than that of the freshwater species in all fishes (about 43\%; Nelson et al. 2016) $\left(\chi^{2}=294, P<0.001\right)$, although phylogenetic distribution of marine and freshwater species should be considered for a sophisticated test of the different likelihood of hermaphroditism evolution between habitats.

Regarding this hermaphroditism absence from most freshwater habitats, it has been suggested that the relatively large eggs or young produced by many freshwater species tend to accentuate the physical differences in male 


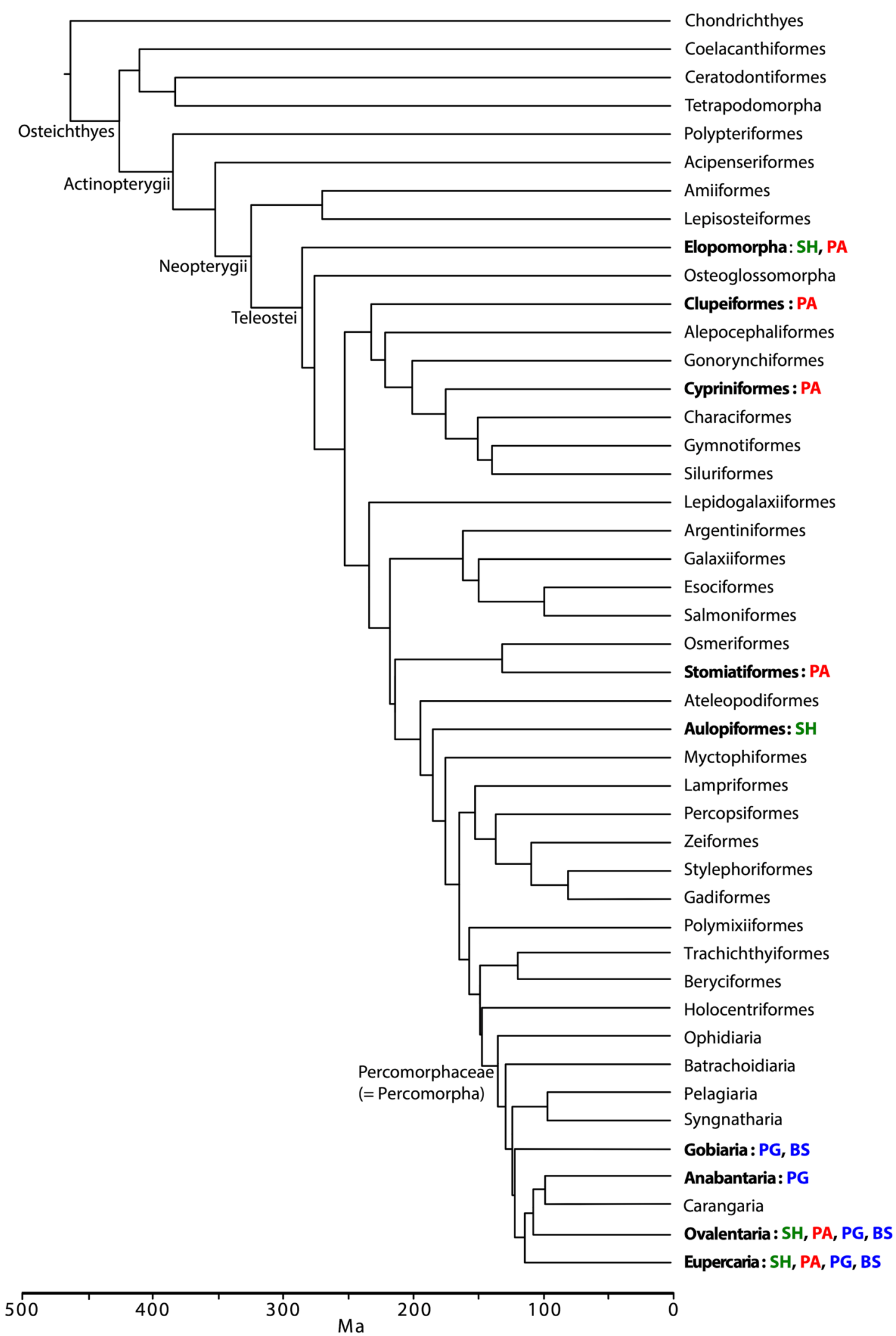

Fig. 1 Phylogeny of fishes and occurrence of hermaphroditism. The phylogenetic tree was processed from Fig. 1 of Betancur-R et al. (2017). $S H$ simultaneous hermaphroditism, $P A$ protandry, $P G$ protog- yny, $B S$ bidirectional sex change or reversed sex change in protogynous species. For group names and orders, see Table 3 
Table 4 Relation between hermaphroditism and mating system. Summed up from Table 1

\begin{tabular}{|c|c|c|c|c|c|c|c|c|c|c|}
\hline \multirow[t]{2}{*}{ Mating system* } & \multicolumn{10}{|c|}{ Number of species in each type of sexual pattern** } \\
\hline & $\mathrm{SH}$ & $\mathrm{SH}, \mathrm{G}$ & PA & PA, G & PG & PG, G & PG, G, BS & PG, BS & $\mathrm{BS}$ & Total \\
\hline Random mating & & & 3 & & & & & & & 3 \\
\hline Non-size-assortative monogamy & & & 10 & & & & & & & 10 \\
\hline Size-assortative monogamy & 9 & & & & 2 & & & 3 & 5 & 19 \\
\hline Harem polygyny & 2 & & & & 45 & 2 & & 4 & 2 & 55 \\
\hline Harem, GSP & & & & & 1 & & & & & 1 \\
\hline Harem, MTV polygamy & & & & & 5 & 3 & 1 & & & 9 \\
\hline Harem, MTV polygamy, GSP & & & & & 1 & & & & & 1 \\
\hline MTV polygamy & & & & & 40 & 1 & & 1 & 2 & 44 \\
\hline MTV polygamy, GSP & & & & & 16 & & & 1 & & 17 \\
\hline Group spawning & & & 1 & & 3 & 2 & & & & 6 \\
\hline Spawning aggregation & & & 1 & 1 & 11 & & & & & 13 \\
\hline Unknown*** & 37 & 7 & 27 & 11 & 150 & 5 & 0 & 8 & 38 & 283 \\
\hline
\end{tabular}

*Harem harem polygyny, MTV polygamy male-territory-visiting polygamy, GSP group spawning

**SH simultaneous hermaphroditism, $P A$ protandry, $P G$ protogyny, $B S$ bidirectional sex change or reversed sex change in protogynous species, $G$ gonochorism

***Mating systems of hermaphroditic species are completely unknown in 26 families (see Table 1) and female anatomy, possibly forming a barrier or physical constraint to hermaphroditism (Warner 1978) and that fewer and larger eggs and more parental care may reduce the size advantage (Sadovy de Mitcheson and Liu 2008). Moreover, mating systems of freshwater fish should also be taken into consideration.

Mating system and the evolution of hermaphroditism. In the field, mating systems have been reported in less than $40 \%$ of hermaphroditic species (Table 4), and most of them were shallow coral reef fishes (Table S1). Mating system is known only from 11 species with SH (20\% of 55 spp.), i.e., small serranids living on coral reefs (Table 1, S1). Reciprocal pair spawning (i.e., egg trading) occurs in monogamous pairs (9 spp., e.g., Fischer 1981), or a large male (secondary male derived from hermaphrodite) controls a harem of simultaneous hermaphrodites (3 spp., e.g., Petersen and Fischer 1986). Mating systems of the other $80 \%$ of species with $\mathrm{SH}$, including deep-sea species (e.g., Aulopiformes), remain unknown. SH in deep-sea fishes are often regarded as evidence for the low-density model (Tomlinson 1966; Ghiselin 1969), because population density is generally low in the deep sea. Although the study of mating behavior in deep-sea fishes is extremely difficult, underwater video tape recordings and the distribution of the number of fish caught have recently suggested pair bonding in two deep-sea SH species of Giganturidae (Kupchik et al. 2018). Another example of hermaphroditism is found in androdioecious (i.e., $\mathrm{SH}$ and males) killifish of genus Kryptolebias, in which hermaphrodites never mate with other hermaphrodites (Furness et al. 2015). but reproduce via selfing or mating with males.

The mating system has only been reported in 16 species with PA (30\% of 54 spp.; Table 4). Non-size-assortative monogamy (i.e., females accept smaller males as in random mating) is known from 10 species of anemonefishes (Pomacentridae; Table 1; e.g., Fricke and Fricke 1977), and random mating from three species of Platycephalidae (Table 1; e.g., Sunobe et al. 2016). These mating systems support the SA model prediction about PA being favored in species with random mating or non-size-assortative monogamy in which females accept smaller males (Warner 1975, 1984). Spawning aggregation and group spawning have also been reported (in two and one species, respectively, of Sparidae; Table 1; e.g., van der Walt and Mann 1998), but whether random mating occurs in the aggregation remains unknown.

Mating systems have been reported from 142 species with PG (47\% of 305 spp.; Table 4). Harem polygyny and male-territory-visiting polygamy (62 and 69 spp., respectively) were the prevailing systems (adding up to $85 \%$ of 142 spp.; Table 4), e.g., Labridae, Scaridae, and Pomacanthidae (Table 1). This supports the SA model prediction of PG being favored in polygynous species with large males monopolizing females (Warner 1975). Size-assortative monogamy has been reported from five species with PG (Table 4). All five species belong to Gobiidae, and BS was also confirmed in three of them (Table 1) and suggested in another one (Table S1). The tendency of PG with BS in the monogamous goby Paragobiodon echinocephalus has been explained by the growth-rate advantage in females (Kuwamura et al. 1994): the difference between sexes in growth rate favors the evolution of sex changer even if the size advantage is the same between the two sexes. For the other exceptional cases of group spawning in two labrid and three serranids, and spawning aggregation in one sparid, two labrids, and nine serranids (Table 1, 4), further observations 
are needed because group spawning is also known in species with male-territory-visiting polygamy (Table 4; Warner and Hoffman 1980; Suzuki et al. 2010).

Mating system has been reported from 19 species with BS (29\% of 66 spp.; Table 4). Size-assortative monogamy is known in eight species (Gobiidae), harem polygyny in seven species (Gobiidae, Pomacentridae, Labridae, Pomacanthidae, and Cirrhitidae), and male-territory-visiting polygamy in five species (Gobiidae, Pomacentridae, and Labridae) (Table 1). BS in the monogamous coral-dwelling gobies has been explained by the risk-of-movement hypothesis (Munday et al. 2010). BS in protogynous and polygynous species can be explained by the low-density hypothesis for the reversed sex change, because facultative monogamy occurs at low-density conditions in polygynous species (Kuwamura et al. 2014, 2016a). Contrastingly, the reversed (female-tomale) sex change does not occur even at very low density in the protandrous anemonefishes (Fricke and Fricke 1977; Kuwamura and Nakashima 1998; T. Kuwamura, unpublished data) or PA species with random mating (Table 1).

Mating system is only known in two of 13 freshwater hermaphroditic species, one in Cichlidae and the other in Synbranchidae (Table 2). Both species are PG with male-territory-visiting polygamy (Table 1), supporting the SA model. Although group spawning is common, mating systems of freshwater gonochorists are as various as in marine species (Taborsky 2008). For example, both monogamy and polygyny are known in cichlid fishes (Kuwamura 1997), but functional hermaphroditism is almost unknown, probably owing to biparental care or prolonged parental care of juveniles, which may reduce the difference in reproductive success between the sexes (Warner and Lejeune 1985).

Effect of population density on mating system and sexual pattern. Intraspecific variations in sexual pattern (including gonochorism) and mating system (excluding facultative monogamy in many polygamous species) have been reported in at least 50 and 28 species, respectively (Tables 1 and 4), which may be partly explained by differences in population density. It is well known that mating system may vary depending on population density (Fricke 1980; Warner and Hoffman 1980; Barlow 1984; Petersen 2006). Accordingly, the low-density hypothesis for the evolution of reversed sex change in polygynous and protogynous species has been proposed and tested (Kuwamura et al. 2002, 2011, 2014, 2016a), predicting that facultative monogamy and reversed sex change may occur under lowdensity condition in polygynous species. For example, in a coral-dwelling damselfish Dascyllus aruanus (Pomacentridae), female-to-male sex change (PG) occurs in harems on isolated corals as the SA model predicts, and reversed sex change (BS) occurs when widowed males meet under experimental low-density conditions at which facultative monogamy occurs (Kuwamura et al. 2016a). In contrast, sex change rarely occurs (i.e., gonochorism) in high-density populations living in continuous coral-covered habitats, where females can choose mates with low risk of movement (Kuwamura et al. 2016a). Moreover, in another pomacentrid, the monogamous and protandrous anemonefish Amphiprion clarkii, sex change rarely occurs in a high-density population with low risk of movement (Ochi 1989).

The effect of population density has also been reported from protogynous labrids. Two types of males (diandry) and alternative male mating tactics are well known in Labridae (Warner and Robertson 1978; Kuwamura et al. 2016b). Primary males mature and remain as males, i.e., gonochorists, and secondary males have changed sex from female (PG). Small IP (initial phase) males participate in group spawning or sneaking, and large TP (terminal phase) males establish mating territories and pair spawn with visiting females. The percentage of IP males (primary males) and the frequency of group spawning increase with population density (Warner and Hoffman 1980; Suzuki et al. 2010), and sex change rarely occurs under high densities (Warner 1982). Contrastingly, reversed (male-to-female) sex change has been reported in low-density populations of Halichoeres trimaculatus (Kuwamura et al. 2007). Reversed sex change in facultatively monogamous pairs in low-density conditions has also been confirmed in a haremic wrasse Labroides dimidiatus (Kuwamura et al. 2014).

These examples suggest that different population densities can cause intraspecific variation in sexual pattern through variations in the mating system. Protogynous species become gonochoristic in high-density conditions and reversed sex change may occur in low-density conditions. In protandrous species; however, reversed sex change has not been reported in low-density conditions and further investigations are needed. In Sparidae, for example, both hermaphroditism (PA or PG) and gonochorism are known in at least 12 species (Table 1, S1). Although their mating systems are almost unknown at present, they will be a good subject to examine the relationship between population density and sexual pattern.

There is much more work to be done to investigate the effects of population density and mating system on interspecific variations in sexual pattern, and we hope that the database presented here should allow for many future comparative tests. To further facilitate this work, we need to create a database of the mating systems of gonochoristic fish species similar to that presented here for hermaphroditic species. We can then proceed to make comparative tests with closely related groups that differ even more strongly in sexual pattern.

Acknowledgments We would like to thank Shohei Suzuki for discussing the early version of a density-dependent sexual pattern hypothesis. 
Our thanks are also due to the anonymous reviewers for constructive comments on the manuscript. This work was supported by JSPS KAKENHI Grant Number 24570033 to T. Kuwamura. We would like to thank Editage (https://www.editage.com) for English language editing.

Open Access This article is licensed under a Creative Commons Attribution 4.0 International License, which permits use, sharing, adaptation, distribution and reproduction in any medium or format, as long as you give appropriate credit to the original author(s) and the source, provide a link to the Creative Commons licence, and indicate if changes were made. The images or other third party material in this article are included in the article's Creative Commons licence, unless indicated otherwise in a credit line to the material. If material is not included in the article's Creative Commons licence and your intended use is not permitted by statutory regulation or exceeds the permitted use, you will need to obtain permission directly from the copyright holder. To view a copy of this licence, visit http://creativecommons.org/licenses/by/4.0/.

\section{References}

Ashman TL, Bachtrog D, Blackmon H, Goldberg EE, Hahn MW, Kirkpatrick M, Kitano J, Mank JE, Mayrose I, Ming R et al (2014) Tree of Sex: A database of sexual systems. Sci Data 1:140015

Avise J (2011) Hermaphroditism: a primer on the biology, ecology, and evolution of dual sexuality. Columbia University Press, New York

Barlow GW (1984) Patterns of monogamy among teleost fishes. Arch FischWiss 35:75-123

Betancur-R R, Wiley EO, Arratia G, Acero A, Bailly N, Miya M, Lecointre G, Orti G (2017) Phylogenetic classification of bony fishes. BMC Evol Biol 17:162

Charnov EL (1982) The theory of sex allocation. Princeton University Press, Princeton

Cole KS, Shapiro DY (1990) Gonad structure and hermaphroditism in the gobiid genus Coryphopterus (Teleostei: Gobiidae). Copeia 1990:996-1003

Devlin RH, Nagahama Y (2002) Sex determination and sex differentiation in fish: an overview of genetic, physiological, and environmental influences. Aquaculture 208:191-364

Erisman BE, Petersen CW, Hastings PA, Warner RR (2013) Phylogenetic perspectives on the evolution of functional hermaphroditism in teleost fishes. Integr Comp Biol 53:736-754

Fischer EA (1981) Sexual allocation in a simultaneously hermaphroditic coral reef fish. Am Nat 117:64-82

Fishelson L (1992) Comparative gonad morphology and sexuality of the Muraenidae (Pisces, Teleostei). Copeia 1992:197-209

Fricke HW (1980) Control of different mating systems in a coral reef fish by one environmental factor. Anim Behav 28:561-569

Fricke H, Fricke S (1977) Monogamy and sex change by aggressive dominance in coral reef fish. Nature 266:830-832

Furness AI, Tatarenkov A, Avise JC (2015) A genetic test for whether pairs of hermaphrodites can cross-fertilize in a selfing killifish. J Hered 106:749-752

Ghiselin MT (1969) The evolution of hermaphroditism among animals. Q Rev Biol 44:189-208

Ghiselin MT (1974) The economy of nature and the evolution of sex. University of California Press, Berkeley, California

Heath DJ (1977) Simultaneous hermaphroditism; cost and benefit. J Theor Biol 64:363-373

Kazancioğlu E and Alonzo SH (2010) A comparative analysis of sex change in Labridae supports the size advantage hypothesis. Evolution 64:2254-2264
Kupchik MJ, Benfield MC, Sutton TT (2018) The first in situ encounter of Gigantura chuni (Giganturidae: Giganturoidei: Aulopiformes: Cyclosquamata: Teleostei), with a preliminary investigation of pair-bonding. Copeia 106:641-645

Kuwamura T (1997) The evolution of parental care and mating systems among Tanganyikan cichlids. In: Kawanabe H, Hori M, Nagoshi M (eds) Fish communities in Lake Tanganyika. Kyoto University Press, Kyoto, pp 57-86

Kuwamura T, Nakashima Y (1998) New aspects of sex change among reef fishes: recent studies in Japan. Environ Biol Fish 52:125-135

Kuwamura T, Kadota T, Suzuki S (2014) Testing the low-density hypothesis for reversed sex change in polygynous fish: experiments in Labroides dimidiatus. Sci Rep 4: 4369

Kuwamura T, Nakashima Y, Yogo Y (1994) Sex change in either direction by growth-rate advantage in the monogamous coral goby, Paragobiodon echinocephalus. Behav Ecol 5:434-438

Kuwamura T, Suzuki S, Kadota T (2011) Reversed sex change by widowed males in polygynous and protogynous fishes: female removal experiments in the field. Naturwissenschaften 98:1041-1048

Kuwamura T, Suzuki S, Kadota T (2016a) Male-to-female sex change in widowed males of the protogynous damselfish, Dascyllus aruanus. J Ethol 34:85-88

Kuwamura T, Suzuki S, Kadota T (2016b) Interspecific variation in the spawning time of labrid fish on a fringing reef at Iriomote Island, Okinawa. Ichthyol Res 63:460-469

Kuwamura T, Suzuki S, Tanaka N, Ouchi E, Karino K, Nakashima Y (2007) Sex change of primary males in a diandric labrid Halichoeres trimaculatus: coexistence of protandry and protogyny within a species. J Fish Biol 70:1898-1906

Kuwamura T, Tanaka N, Nakashima Y, Karino K, Sakai Y (2002) Reversed sex-change in the protogynous reef fish Labroides dimidiatus. Ethology 108:443-450

Manabe H, Ishimura M, Shinomiya A, Sunobe T (2007) Field evidence for bi-directional sex change in the polygynous gobiid fish Trimma okinawae. J Fish Biol 70:600-609

Mank JE, Promislow DE, Avise JC (2006) Evolution of alternative sex-determining mechanisms in teleost fishes. Biol J Linn Soc 87:83-93

Munday PL, Buston PM, Warner RR (2006) Diversity and flexibility of sex-change strategies in animals. Trends Ecol Evol 21:89-95

Munday PL, Kuwamura T, Kroon FJ (2010) Bidirectional sex change in marine fishes. In: Cole KS (ed) Reproduction and sexuality in marine fishes: patterns and processes. University of California Press, Berkeley, California, pp 241-271

Muñoz RC, Warner RR (2003) A new version of the size-advantage hypothesis for sex change: incorporating sperm competition and size-fecundity skew. Am Nat 161:749-761

Muñoz RC, Warner RR (2004) Testing a new version of the size-advantage hypothesis for sex change: sperm competition and size-skew effects in the bucktooth parrotfish, Sparisoma radians. Behav Ecol 15:129-136

Nakazono A, Kuwamura T (eds) (1987) Sex change in fishes. Tokai University Press, Tokyo

Nelson JS (2006) Fishes of the world, 4th edn. John Wiley \& Sons, New York

Nelson JS, Grande TC, Wilson MVH (2016) Fishes of the world, 5th edn. John Wiley \& Sons, New Jersey

Ochi H (1989) Mating behavior and sex change of the anemonefish, Amphiprion clarkii, in the temperate waters of southern Japan. Environ Biol Fish 26:257-275

Petersen CW (2006) Sexual selection and reproductive success in hermaphroditic seabasses. Integr Comp Biol 46:439-448 
Petersen CW, Fischer EA (1986) Mating system of the hermaphroditic coral-reef fish, Serranus baldwini. Behav Ecol Sociobiol 19:171-178

Sadovy Y, Shapiro DY (1987) Criteria for the diagnosis of hermaphroditism in fishes. Copeia 1987:136-156

Sadovy de Mitcheson Y, Liu M (2008) Functional hermaphroditism in teleosts. Fish Fish (Oxf) 9:1-43

Sunobe T, Sakaida S, Kuwamura T (2016) Random mating and protandrous sex change of the platycephalid fish Thysanophrys celebica (Platycephalidae). J Ethol 34:15-21

Sunobe T, Sado T, Hagiwara K, Manabe H, Suzuki T, Kobayashi Y, Sakurai M, Dewa S, Matsuoka M, Shinomiya A, Fukuda K, Miya M (2017) Evolution of bidirectional sex change and gonochorism in fishes of the gobiid genera Trimma, Priolepis, and Trimmatom. Sci Nat 104:15

Suzuki S, Kuwamura T, Nakashima Y, Karino K, Kohda M (2010) Social factors of group spawning as an alternative mating tactic in the territorial males of the threespot wrasse Halichoeres trimaculatus. Environ Biol Fish 89:71-77

Taborsky M (2008) Alternative reproductive tactics in fish. In: Oliveira RF, Taborsky M, Brockmann HJ (eds) Alternative reproductive tactics: an integrative approach. Cambridge University Press, Cambridge, pp 251-299

Tomlinson J (1966) The advantages of hermaphroditism and parthenogenesis. J Theor Biol 11:54-58

Van der Walt BA, Mann BQ (1998) Aspects of the reproductive biology of Sarpa salpa (Pisces: Sparidae) off the east coast of South Africa. Afr Zool 33:241-248
Warner RR (1975) The adaptive significance of sequential hermaphroditism in animals. Am Nat 109:61-82

Warner RR (1978) The evolution of hermaphroditism and unisexuality in aquatic and terrestrial vertebrates. In: Reese ES, Lighter FJ (eds) Contrasts in behavior. John Wiley \& Sons, New York, pp 77-101

Warner RR (1982) Mating systems, sex change and sexual demography in the rainbow wrasse, Thalassoma lucasanum. Copeia 1982:653-661

Warner RR (1984) Mating behavior and hermaphroditism in coral reef fishes. Am Sci 72:128-136

Warner RR (1988) Sex change in fishes: hypotheses, evidence, and objections. Environ Biol Fish 22:81-90

Warner RR, Hoffman SG (1980) Local population size as a determinant of mating system and sexual composition in two tropical marine fishes (Thalassoma spp.). Evolution 34:508-518

Warner RR, Lejeune P (1985) Sex change limited by paternal care: a test using four Mediterranean labrid fishes, genus Symphodus. Mar Biol 87:89-99

Warner RR, Robertson DR (1978) Sexual patterns in the labroid fishes of the Western Caribbean. II: The wrasses (Labridae). Smithson Contrib Zool 254:1-27

Publisher's Note Springer Nature remains neutral with regard to jurisdictional claims in published maps and institutional affiliations. 